\title{
Exact model of the power-to-efficiency trade-off while approaching the Carnot limit
}

\author{
Clifford V. Johnson* \\ Department of Physics and Astronomy University of Southern California Los Angeles, \\ California 90089-0484, USA
}

(Received 14 April 2017; published 6 July 2018)

\begin{abstract}
The Carnot heat engine sets an upper bound on the efficiency of a heat engine. As an ideal, reversible engine, a single cycle must be performed in infinite time, and so the Carnot engine has zero power. However, there is nothing, in principle, forbidding the existence of a heat engine whose efficiency approaches that of Carnot while maintaining finite power. Such an engine must have very special properties, some of which have been discussed in the literature, in various limits. While recent theorems rule out a large class of engines from maintaining finite power at exactly the Carnot efficiency, the approach to the limit still merits close study. Presented here is an exactly solvable model of such an approach that may serve as a laboratory for exploration of the underlying mechanisms. The equations of state have their origins in the extended thermodynamics of electrically charged black holes.
\end{abstract}

DOI: 10.1103/PhysRevD.98.026008

It is well known that a heat engine, regardless of working substance and the details of the thermodynamic cycle, has a fundamental limit on its efficiency given by the Carnot efficiency $\eta_{\mathrm{C}}$. If $T_{H}$ is the highest operating temperature in the engine and $T_{C}$ the lowest (the temperatures at which the input heat $Q_{H}$ and exhaust heat $Q_{C}$ are exchanged with the hot and cold reservoirs, respectively), the efficiency $\eta$ is bounded as follows:

$$
\eta=1-\frac{Q_{C}}{Q_{H}} \leq \eta_{\mathrm{C}}=1-\frac{T_{C}}{T_{H}} .
$$

It is also familiar that the Carnot engine itself is an idealized reversible engine, with a cycle that is composed of two isotherms and two adiabats, with the expansions and compressions performed quasistatically, in order to maintain reversibility. In other words, it takes an infinite amount of time to perform one cycle of the Carnot engine: It has zero power.

While most typical heat engines, working at finite power, operate well below the Carnot efficiency, there is no issue of principle that prevents their efficiency from approaching that of Carnot, but it becomes increasingly difficult for typical working substances and choices of operating cycle. The question naturally arises as to what kind of engine is

\footnotetext{
*johnson1@usc.edu
}

Published by the American Physical Society under the terms of the Creative Commons Attribution 4.0 International license. Further distribution of this work must maintain attribution to the author(s) and the published article's title, journal citation, and DOI. Funded by SCOAP ${ }^{3}$. needed to approach the Carnot efficiency while maintaining finite power. (This is a separate issue from the CurzonAhlborn bound on $\eta$ when working at maximum power [1].) There have been recent discussions of this in the thermodynamics and statistical mechanics literature [2-10], and two papers in particular [6,7] consider having the working substance near criticality as an approach to the problem, exploiting either fluctuations, or a diverging heat capacity to argue for the maintenance of finite power as $\eta$ grows closer to $\eta_{\mathrm{C}}$. It has been argued in Refs. $[9,10]$ that it is forbidden (for widely applicable assumptions) to be exactly at the Carnot efficiency while at finite power, but the issue of the approach to the limit is still of considerable interest, for both practical and theoretical reasons. This paper presents an exactly solvable model of such an approach that may be of use in gaining better understanding of how various models (perhaps less computationally accessible) work. A critical system will also feature in the present work, but its role appears to be of quite a different character from what was argued for in the systems of Refs. [6,7]. Fluctuations and diverging specific heat do not explicitly play an essential role in the core construction. This can be determined because the system employed can be readily queried with a computation: All the needed properties of the working substance are available via a full set of exact defining equations.

The system to be used here has its origins in extended gravitational thermodynamics: The traditional treatment [11] of black holes in semiclassical quantum gravity supplies them with a temperature $T$ and an entropy $S$, which depend upon the mass $M$ and the horizon radius $r_{+}$. This treatment is extended [12] by making dynamical the 
cosmological constant $(\Lambda)$ of the gravity theory [13], which yields a pressure variable $p=-\Lambda / 8 \pi$ [15] and its conjugate volume $V \equiv(\partial H / \partial p)_{S}$. The enthalpy $H$ is the black hole's mass, and the first law in terms of all these quantities is $d H=T d S+V d p$. Studies of the extended thermodynamics of gravitational systems have uncovered many phenomena that are familiar from statistical physics and thermodynamics. (For a recent review, see Ref. [16].)

Notice that for negative $\Lambda$ the pressure is positive. Reference [17] presented the idea of defining heat engines that do traditional mechanical work $W=\int p d V$ in this context [18]. The heat flows $Q_{H}$ and $Q_{C}$ into and out of the engine can be considered as from and to nonbackreacting heat baths of radiation filling the spacetime, as is traditional in black hole thermodynamics. (See, e.g., Ref. [19].) Such engines were called holographic heat engines since gravitational physics in spacetimes with negative $\Lambda$ is known to have a dual description in terms of strongly coupled nongravitational physics (in one dimension fewer). These are examples of a broader phenomenon in quantum gravity known as holography. (For a review, see Ref. [14]). Such dualities are not needed here, but it is worth noting that if the gravitational language is not to the reader's taste, it could, in principle, be translated to the language of a class of strongly coupled gauge theories. In other words, the gravitational aspect of this example is not essential, but it is more economical to use that simpler language.

The context will be a $(3+1)$-dimensional EinsteinMaxwell system with action

$$
I=-\frac{1}{16 \pi} \int d^{4} x \sqrt{-g}\left(R-2 \Lambda-F^{2}\right)
$$

where $\Lambda=-3 / l^{2}$ sets a length scale $l$. The black hole spacetimes of interest here are Reissner-Nordstrom-like solutions of charge $q$. The metric and gauge potential are

$d s^{2}=-Y(r) d t^{2}+\frac{d r^{2}}{Y(r)}+r^{2}\left(d \theta^{2}+\sin ^{2} \theta d \varphi^{2}\right)$,

$Y(r) \equiv 1-\frac{2 M}{r}+\frac{q^{2}}{r^{2}}+\frac{r^{2}}{l^{2}}, \quad A_{t}=q\left(\frac{1}{r_{+}}-\frac{1}{r}\right)$.

The potential is chosen to vanish on the horizon at $r=r_{+}$, the largest positive real root of $Y(r)$.

Several aspects of the thermodynamics of these solutions were studied in Refs. [20,21]. There, a rich phase structure was uncovered, a van der Waals-like nature was elucidated, including a second-order critical point. By including variable $\Lambda$ (and, hence, a pressure $p$ ), Ref. [22] clarified the resemblance to van der Waals and showed that the system has the same universal behavior near the critical point as the van der Waals gas.

The standard semiclassical quantum gravity procedures [11] yield a temperature for each black hole solution, which depends on $r_{+}, q$, and $\Lambda$. The entropy is given by a quarter of the area of the horizon: $S=\pi r_{+}^{2}$. The extended thermodynamics [12] allows for all appearances of the length scale $l$ to be replaced by the pressure $p$ using the relation $p=3 /\left(8 \pi l^{2}\right)$, and the thermodynamic volume turns out to be $V=4 \pi r_{+}^{3} / 3$. So all occurrences of $r_{+}$can be traded in for either an $S$ or a $V$, as they are not independent. All of this results in an equation of state $p(V, T)$ :

$$
p=\frac{1}{8 \pi}\left(\frac{4 \pi}{3}\right)^{\frac{4}{3}}\left(\frac{3 T}{V^{\frac{1}{3}}}-\left(\frac{3}{4 \pi}\right)^{\frac{2}{3}} \frac{1}{V^{\frac{2}{3}}}+\frac{q^{2}}{V^{\frac{4}{3}}}\right) .
$$

Some sample isotherms are plotted in Fig. 1. Note that there is a wedge-shaped exclusion region extending from the $V=0$ axis, bounded on the right by the $T=0$ curve (the dashed line) and on the bottom by the $p=0$ line. Points inside that region are unphysical, having $T<0$. (The black hole at $(T=0, p=0)$ is the extremal Reissner-Nordstrom black hole of volume $V_{0}=4 \pi q^{3} / 3$.) Below a critical isotherm the isotherms yield multiple values for $p$, and (in full parallel with the classic van der Waals system) are "repaired" by an isobar (not shown in Fig. 1) at a value of the pressure determined by a study of the free energy. This results in a family of first-order phase transitions between large and small black holes terminating in a second-order critical point at the critical isotherm $[20,21]$. The details of the first-order transitions will not affect the main issue being addressed here, and so they won't be explored further.

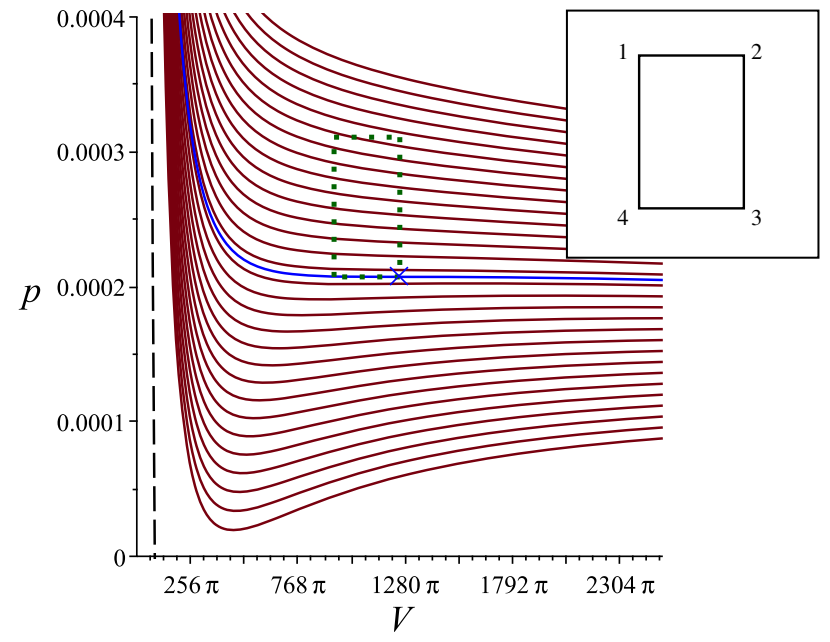

FIG. 1. Main: Sample isotherms for $q=4$. The temperature is higher for the curves further away from the origin. The central (blue) isotherm is at the critical temperature, and the (blue) cross marks the critical point. The isotherms at lower temperatures get modified, as discussed in the main text, but this is not shown here. The dotted green rectangle is an example of the special engine cycle discussed in the text (with $L=1$ ). The dashed curve is the $T=0$ isotherm. Inset: The labeling of the engine cycle. 
An equivalent expression to Eq. (4) is

$$
T=\frac{1}{4 \sqrt{\pi}} S^{-\frac{3}{2}}\left(8 p S^{2}+S-\pi q^{2}\right) .
$$

Meanwhile, the mass (enthalpy) $H(S, p)$ is [23]

$$
M \equiv H=\frac{1}{6 \sqrt{\pi}} S^{-\frac{1}{2}}\left(8 p S^{2}+3 S+3 \pi q^{2}\right),
$$

and the constant $V$ and $p$ specific heats are [22]

$$
C_{V}=0 ; \quad C_{p}=2 S\left(\frac{8 p S^{2}+S-\pi q^{2}}{8 p S^{2}-S+3 \pi q^{2}}\right) .
$$

It is these black holes that were the working substance in the prototype holographic heat engine of Ref. [17], using a rectangular cycle in the $(p, V)$ plane made of isobars and adiabats (which are equivalent to isochors for static black holes since $S$ and $V$ both depend only on $r_{+}$). The inset of Fig. 1 shows the labeling of the cycle to be used below. Later, in Ref. [24], an exact equation for the efficiency was derived for the cycle, and it will be extremely useful here. Key is that the heat flows can be written as mass/enthalpy differences, giving

$$
\eta=1-\frac{Q_{C}}{Q_{H}}=1-\frac{M_{3}-M_{4}}{M_{2}-M_{1}},
$$

where $M_{i}$ is the black hole mass evaluated at the $i$ th corner. Its simplicity means that there is no need to make the kinds of approximations (e.g., high temperature or pressure) usually needed to write explicit efficiency formulae for some particular choice of location of this cycle in the $(p, V)$ plane.

The next step is to decide where to place the cycle in the $(p, V)$ plane. In previous work in this area, $q$ has been treated essentially as a label for a family of solutions, and was conveniently set to a positive nonzero value and forgotten about, since the key features don't depend upon its actual value. This will not be the case here. Consider making $q$ large, for reasons that will become clear shortly. For a given choice of the position of the cycle [choosing a range for $p$ and $V($ or $S$ )], a sensible engine can be defined, but for large enough $q$ eventually the system will become unphysical: $T$ [see Eq. (5)] on some parts of the cycle starts becoming negative because the exclusion region moves to the right with increasing $q$. This can be avoided by seeking choices for the $p, V$ (or $S$ ) coordinates of the cycle variables that scale with $q$ in such a way as to stay physical. Equation (5) shows that the scaling is $S \sim q^{2}, p \sim q^{-2}$, and hence $T \sim q^{-1}$. There is a very distinguished point exhibiting this exact scaling behavior. It is the second-order critical point, defined by the $p(V, T)$ curve with a point of inflection: $\partial p / \partial V=\partial^{2} p / \partial V^{2}=0$, $p_{\mathrm{cr}}=\frac{1}{96 \pi q^{2}}, \quad S_{\mathrm{cr}}=6 \pi q^{2}, \quad T_{\mathrm{cr}}=\frac{1}{3 \sqrt{6} \pi q}$,

with $V_{\text {cr }}=8 \sqrt{6} \pi q^{3}$. (See Fig. 1 for the case of $q=4$.)

So if the engine cycle is chosen to be in the neighborhood of this point, and of a size that does not extend into the exclusion region, it will be physical. There are several ways of making such a choice, and one family will be chosen here for illustration. Place the critical point at corner 3: $p_{3}=p_{4}=p_{\mathrm{cr}}$, and choose the upper isobar [25] to be at some multiple of $p_{\mathrm{cr}}: p_{1}=p_{2}=3 p_{\mathrm{cr}} / 2$. (See the dotted rectangle in Fig. 1.) In preparation for large $q$, it is to be noted that since $p \sim q^{-2}$, the cycle is in danger of shrinking to zero area, giving vanishing work and hence vanishing $\eta$. But if $V_{2}-V_{1}=V_{3}-V_{4}$ are chosen to scale as $q^{2}$, then the work will be finite at any $q$. So $V_{2}=V_{3}=V_{\text {cr }}$ while $V_{1}=V_{4}=V_{\text {cr }}-V_{\text {cr }} L / q$, where $L$ is a constant. This gives $W=p_{\mathrm{cr}} V_{\mathrm{cr}} L / 2 q=L / 4 \sqrt{6}$. So as $q$ is increased, the whole cycle shrinks vertically, but grows horizontally in such a way as to keep the work done finite. It is now a matter of studying the $q$ dependence of the input heat $Q_{H}$. It is simply the mass (enthalpy) difference $M_{2}-M_{1}$, with $p=3 p_{\mathrm{cr}} / 2, S_{2}=S_{\mathrm{cr}}$ and $S_{1}=S_{\mathrm{cr}}(1-L / q)^{\frac{2}{3}}$ placed into Eq. (6). Interestingly, the large $q$ expansion of $Q_{H}$ decreases to a limiting value,

$$
Q_{H}=\frac{19 \sqrt{6}}{72} L+\frac{\sqrt{6}}{27} \frac{L^{2}}{q}+\frac{4 L^{3} \sqrt{6}}{243 q^{2}}+O\left(q^{-3}\right),
$$

and hence the efficiency $\eta=W / Q_{H}$ is, at large $q$,

$\eta=\frac{3}{19}-\frac{8}{361} \frac{L}{q}-\frac{416 L^{2}}{61731 q^{2}}-\frac{3286 L^{3}}{1172889 q^{3}}+O\left(q^{-4}\right)$.

The next thing to do is compute the Carnot efficiency for the engine. Directly inserting the chosen values for $\left(S_{2}, p_{2}\right)$ and $\left(S_{4}, p_{4}\right)$ into Eq. (5) gives $T_{H}=(19 \sqrt{6} / 288)(\pi q)^{-1}$ exactly, while the large $q$ expansion for $T_{C}$ begins

$$
T_{C}=\frac{1}{18} \frac{\sqrt{6}}{\pi q}-\frac{L^{3} \sqrt{6}}{972 \pi q^{4}}-\frac{7 L^{4} \sqrt{6}}{3888 \pi q^{5}}+O\left(q^{-6}\right),
$$

and so,

$$
\eta_{\mathrm{C}}=1-\frac{T_{C}}{T_{H}}=\frac{3}{19}+\frac{8 L^{3}}{513 q^{3}}+\frac{14 L^{4}}{513 q^{4}}+O\left(q^{-5}\right) .
$$

These simple but striking results constitute the main demonstration promised for this paper. (Figure 2 is a plot of the ratio $\eta / \eta_{\mathrm{C}}$ vs $q$, showing the rise to unity at large $q$.) This is a heat engine that does finite work at any $q$, and $\eta \rightarrow \eta_{\mathrm{C}}$ as $q \rightarrow \infty$. This is atypical, since usually going to the Carnot limit for one of the classic heat engine cycles (or variants thereof) translates into vanishing or infinite work. 


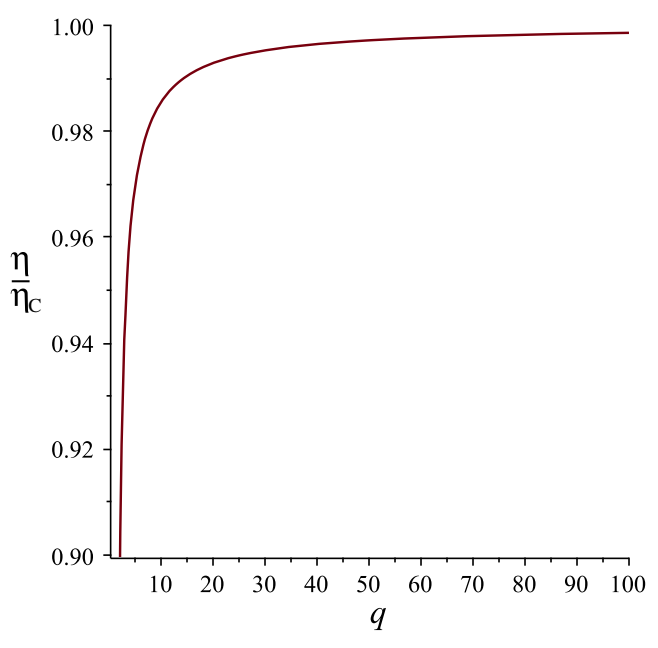

FIG. 2. The behavior of the ratio $\eta / \eta_{\mathrm{C}}$ as a function of $q$ for the prototype scheme. Variations of the scheme discussed in the text behave qualitatively similarly.

As an example, the Otto cycle has $\eta=1-r^{1-\gamma}$, where $\gamma=$ $C_{p} / C_{V}>1$ and $r$ is the ratio of largest to smallest volumes, and so $\eta$ is maximized for $r \rightarrow \infty$. As another, the (Braytonlike) rectangular cycle defined for black holes at high pressures and temperatures well away from the critical point has [17] $\eta=\left(1-p_{4} / p_{1}\right)\left(1+O\left(1 / p_{1}\right) \cdots\right)$ (using the labeling in Fig. 1), which may be written as

$$
\eta=1-\frac{T_{C}}{T_{H}}\left(\frac{V_{2}}{V_{4}}\right)^{1 / 3}+\cdots
$$

This is an analogue of an ideal gas limit [26], and Carnot efficiency is approached if $V_{2} \rightarrow V_{4}$, resulting in no work. In the case under consideration however, the region of interest is far from an ideal gas regime and in fact as $q$ grows $T$ decreases. This is appealing since most of the current discussions in the literature about physical realizations of finite power efficient engines are about low temperature experiments. Finite work here as $\eta \rightarrow \eta_{\mathrm{C}}$ is a useful feature to have under control on the way to studying finite power.

The time taken to do a cycle, $\tau$, is all that needs to be examined next. On the face of it, that seems to be finite at any finite $q$ (but see further discussion below) and so this is indeed a model of an approach to Carnot while maintaining finite power in the following precise sense: An efficiency as close to the Carnot efficiency as desired can be achieved by choosing large enough $q$, and picking the cycle according to the prescription above. Precisely at the limit $\eta=\eta_{\mathrm{C}}$, however, while the work is finite, the power has vanished, since the pressures in the isobars are proportional to $p_{\mathrm{cr}} \sim q^{-2}$, which vanishes in the limit, meaning that the time it takes to perform the isobaric expansions and compressions diverges. So the inequality of, e.g., Refs. $[9,10]$ showing the unattainability of finite power exactly at Carnot efficiency is easily satisfied. It is

$$
\frac{W}{\tau} \leq \bar{\Theta} \frac{\eta\left(\eta_{\mathrm{C}}-\eta\right)}{T_{C}}
$$

where $\bar{\Theta}$ is a model-dependent constant capturing the characteristics of the engine. For the current model, the right hand side (divided by $\bar{\Theta}$ ) has a large $q$ expansion that begins as: $72 \pi \sqrt{6} L / 6859+224 \pi \sqrt{6} L^{2} /(130321 q)+$ $O\left(q^{-2}\right)$. Meanwhile, the work $W=L / 4 \sqrt{6}$, but an optimistic estimate of the $q$ dependence of $\tau$ based on the behavior of the pressures (discussed above) is $\tau \sim q^{2}$.

The above cycle is just one example of the kind of scheme that will work. Variations were studied, and some are worth reporting the results for. One is to put the critical point at a different point on the lower isobar. This results in qualitatively similar results at large $q$. The difference is that both $T_{H}$ and $T_{C}$ have higher-order corrections to their leading $1 / q$ form at large $q$. Again, $\eta$ and $\eta_{\mathrm{C}}$ converge at large $q$ to $3 / 19$. It should also be noted that the approach $\eta \rightarrow \eta_{\mathrm{C}}$ at large $q$ is achieved even if the critical point is not anywhere on the cycle itself. It suffices to be near enough to the critical region, in the manner outlined.

A concern that might be raised is whether the presence of a critical point somewhere on the cycle might invalidate the claim to be able to achieve finite power (at large but finite $q$ ) due to possible critical slowing of the system. As mentioned above, similar results were achieved by avoiding the critical point, only having the cycle be near it, with $\eta$ approaching $\eta_{\mathrm{C}}$ at large $q$ as before. So the critical point's presence directly on the cycle is not crucial. There might have been an expectation that certain aspects of the physics near the critical point itself are responsible for the (finite power) approach to the Carnot efficiency at large $q$. For example, in the discussion in Ref. [7], it is argued that the divergence of the specific heat produces an enhancement in the power's scaling with the effective system size, $N$, through an enhancement in the heat flows. (There are $N$ coupled quantum Otto engines constituting the system.) Here, $q$ acts as a system size parameter analogous to that paper's $N$. Indeed, near criticality $C_{p}$ 's peak (inverse) width and height are enhanced with increasing $q$, as happens in Ref. [7]. But the explicit expressions for $Q_{H}$ and $Q_{C}=$ $W-Q_{H}$ show that they actually decrease with increasing $q$, even with the critical point on the isobar. Also, the fact that the same qualitative behavior happens away from the critical point suggests that in this model the peak in $C_{p}$ plays no crucial role in driving the efficiency toward Carnot. On the other hand, since the construction presented here removes the $q$ dependence (analogously, $N$ dependence) from the work and places it all into the heat flows, it is difficult to compare the approaches further.

Nevertheless, the critical point itself is important in the whole scheme, since as shown, its neigborhood [which depends on $q$, see Eq. (9)] is key in determining the coordinates of the cycle needed to approach the Carnot efficiency as $q$ increases. A qualitative reason why this all 
works so well is as follows: The neighborhood of the critical point on the critical isotherm, being a region containing a point of inflection, is locally quite horizontal. Other isotherms in the region will inherit some of this behavior, and this is even more true at higher $q$. Close to horizontal means that they do not deviate too far from the isobar shape of the 1-2 and 3-4 parts of the cycle. As discussed earlier, the vertical 2-3 and 4-1 isochoric parts are also adiabats (because of the properties of static black holes). So a prescription for picking a cycle that stays in the neighborhood of the critical point therefore ensures that the cycle itself becomes an increasingly better approximation to a Carnot cycle (two isotherms and two adiabats) as $q$ grows. The behavior of the pressures resulting from this is such that they will vanish in the limit and result in diverging $\tau$ as expected for Carnot.

The underlying system controlling the physics at large $q$ is worth further investigation: It has low pressure and temperature, and high volume and entropy. In the gravitational model it originates as a special family of large charge black holes, but there might be analogues of such equations of state in other, nongravitational, systems. They would be interesting to identify.

\section{ACKNOWLEDGMENTS}

C. V. J. thanks the U.S. Department of Energy for support under Grant No. DE-FG03-84ER-40168 and the Simons Foundation for a Simons Fellowship No. 395705 and Amelia for her support and patience.
[1] F. L. Curzon and B. Ahlborn, Am. J. Phys. 43, 22 (1975).

[2] G. Benenti, K. Saito, and G. Casati, Phys. Rev. Lett. 106, 230602 (2011).

[3] A. E. Allahverdyan, K. V. Hovhannisyan, A. V. Melkikh, and S. G. Gevorkian, Phys. Rev. Lett. 111, 050601 (2013).

[4] K. Proesmans and C. Van den Broeck, Phys. Rev. Lett. 115, 090601 (2015).

[5] K. Brandner, K. Saito, and U. Seifert, Phys. Rev. X 5, 031019 (2015).

[6] M. Polettini, G. Verley, and M. Esposito, Phys. Rev. Lett. 114, 050601 (2015).

[7] M. Campisi and R. Fazio, Nat. Commun. 7, 11895 (2016).

[8] J. Koning and J. O. Indekeu, Eur. Phys. J. B 89, 248 (2016).

[9] N. Shiraishi, K. Saito, and H. Tasaki, Phys. Rev. Lett. 117, 190601 (2016).

[10] N. Shiraishi and H. Tajima, Phys. Rev. E 96, 022138 (2017).

[11] G. W. Gibbons and S. W. Hawking, Phys. Rev. D 15, 2752 (1977).

[12] D. Kastor, S. Ray, and J. Traschen, Classical Quantum Gravity 26, 195011 (2009).

[13] A dynamical $\Lambda$ can be naturally implemented if the gravity theory is embedded within a theory with other dynamical sectors. An example is when there are dynamical scalars $\varphi_{i}$ with a potential $V\left(\varphi_{i}\right)$. Moving between fixed points of the potential, where the scalars take on fixed values $\varphi_{i}^{\mathrm{c}}$, that set a nonzero constant $V\left(\varphi_{i}^{\mathrm{c}}\right)$, setting the value of $\Lambda$. See, e.g., the review in Ref. [14].

[14] O. Aharony, S. S. Gubser, J. M. Maldacena, H. Ooguri, and Y. Oz, Phys. Rep. 323, 183 (2000).
[15] Here we are using geometrical units where $G, c, \hbar, k_{\mathrm{B}}$ have been set to unity.

[16] D. Kubiznak, R. B. Mann, and M. Teo, Classical Quantum Gravity 34, 063001 (2017).

[17] C. V. Johnson, Classical Quantum Gravity 31, 205002 (2014).

[18] Work can be interpreted here results in a change of the overall energy of a spacetime since the volume removes portions of it from the standard energy integral. Recall that $p$ sets an energy density via $\Lambda$ 's equation of state $\rho=-p$ and so a positive change $d V$ results in an energy gain $|\rho| d V$. See Ref. [12].

[19] S. W. Hawking and D. N. Page, Commun. Math. Phys. 87, 577 (1983).

[20] A. Chamblin, R. Emparan, C. V. Johnson, and R. C. Myers, Phys. Rev. D 60, 064018 (1999).

[21] A. Chamblin, R. Emparan, C. V. Johnson, and R. C. Myers, Phys. Rev. D 60, 104026 (1999).

[22] D. Kubiznak and R. B. Mann, J. High Energy Phys. 07 (2012) 033.

[23] B. P. Dolan, Classical Quantum Gravity 28, 235017 (2011).

[24] C. V. Johnson, Entropy 18, 120 (2016).

[25] The choice of placing the critical point on the top isobar can also be made. The cycle dips into the repaired region where there are first-order transitions. However, it is easier to determine the temperatures on the isobars away from that region, and so for the sake of simplicity, the bottom isobar was chosen.

[26] C. V. Johnson, Classical Quantum Gravity 33, 215009 (2016). 